\title{
A classificação de risco como linguagem da rede de urgência e emergência
}

\author{
Welfane Cordeiro Júnior \\ urgencia@saude.mag.gov.br \\ welcj@uai.com.br
}

\section{RESUMO}

Um sistema integrado de atenção às urgências atual deve ter como bases estruturais:

1) Um sistema de classificação de risco único;

2) Fluxos pactuados e bem estabelecidos internamente nos pronto-socorros e externamente em todos os pontos de atenção e logística do sistema;

3) Comando único de um complexo regulador;

4) Qualificação adequada das equipes, de acordo com a responsabilidade da instituição que se estrutura com base em economia de escala e acesso na resposta da rede. A economia de escala é na área da saúde diretamente proporcional à qualidade da resposta.

Ou seja, o paciente certo, no local certo, no tempo certo é a receita adequada para se salvar vidas e também eficientizar o uso dos recursos.

Palavras chaves: redes, triagem, urgência e emergência

No final da década de 80, quando nascia constitucionalmente o SUS, alguns dos países que já haviam universalizado seus sistemas de saúde como o Canadá e o Reino Unido, iniciaram o que foi chamado pelos estudiosos de sistemas de saúde de terceira geração de reformas sanitárias, que propunham uma radical inversão do modelo de atenção à saúde, aprofundando a tendência de reforço do primeiro nível de atenção, como sustentação de uma estratégia que redefinia a vinculação dos pacientes no sistema, não mais nos locais de atenção de pacientes agudos, como os hospitais e sim num acompanhamento horizontal e de longo prazo por parte, principalmente, de uma equipe multidisciplinar no primeiro nível de atenção. Paralelamente, no início da década de 90 ocorre a maior revolução tecnológica da história da humanidade, com a expansão assustadora de um meio de comunicação que forçou e tem forçado um rearranjo de todas as formas de organizações humanas: a internet. O sociólogo Manuel Castells, que escreveu a trilogia "A Era da Informação" durante a década de 90, aponta no primeiro livro ("A sociedade em rede") que o choque que o advento da internet provocou, mudou, praticamente, todas as formas econômicas de produção e relações da sociedade, causando uma transformação irreversível dos modos convencionais de inserção cultural, econômica e social no mundo. Castells postula que daqui prá frente (estamos em plena transição) é praticamente impossível em qualquer ramo da atividade humana não nos organizarmos em rede. Mas a transição é dolorosa para a maioria da sociedade. Nosso olhar ainda é totalmente estabelecido por um modelo cultural nascido há mais de 300 anos e que se tornou vitorioso sobre a obscuridade religiosa da idade média e é também o grande responsável pela evolução tecnológica da humanidade. Este modelo, que alguns chamam de mecanicista em decorrência das metáforas elaboradas pelos grandes cientistas da época, como lsaac Newton, que enxergavam o universo como uma máquina perfeita e previsível, baseouse em 3 grandes princípios:

1) ordem: o universo é ordenado e previsível e o acaso deve ser descartado. Laplace, o Newton francês, postulava que um demônio que conhecesse as leis do universo poderia prever todas as ações do futuro.

2) separação: para estudar um fenômeno é preciso separá-lo de seu meio. As disciplinas também teriam que ser separadas. O observador do fenômeno, naturalmente, também é separado deste. 
3) Razão: A obediência às regras clássicas, não apenas da dedução e indução, mas também dos princípios da contradição, da identidade e do terceiro excluído.

Este modelo levou-nos a grandes avanços tecnológicos e sociais derivando dele toda a estrutura política, social e econômica que vivenciamos no último século. Ocorre que final do século XIX e no início do século XX começam a surgir estudos sobre os quais a base clássica da ciência é impotente para dar explicações. O exemplo mais conhecido é o da mecânica quântica, na física. O universo não seria tão ordenado como se supunha e a incerteza é uma variável importante.

Os estudos que possibilitaram o surgimento da ecologia no meio do século passado têm dificultado a utilização do princípio clássico da separação. É o fim da inocência da ciência clássica, e da compreensão dos fenômenos naturais separando-os de seu contexto, ou seja, sem levar em conta as conexões entre os fenômenos. E por fim, a razão que é um belo instrumento para determinadas situações, limita-se a alguns contextos como nos mostra a frase de Tarski: "Nenhum sistema dispõe de meios suficientes para se explicar a si próprio". Freud e a psicanálise mostraram-nos o quanto as variáveis irracionais eram importantes.

E o que isso tem a ver com nosso texto?

Observem que no momento em que começamos a ter que trabalhar em redes, ou seja conectados uns aos outros, toda a base teórica de nossa formação é limitante. Somos ainda formados e organizados socialmente em um modelo que não consegue lidar com conexões.

O educador e filósofo francês Edgard Morin que coordenou uma reforma no modelo de educação em seu país propõe que nos preparemos e ensinemos as pessoas a lidar com a "complexidade", que hoje tem sido a palavra que sintetiza toda esta revolução, neste novo modelo científico.

De fato e voltando ao nosso tema, a discussão de redes na área de saúde é antiga, mas, de 10 anos para cá o tema tem crescido de forma exponencial na literatura. O que tem pressionado os gestores de sistemas de saúde à discussão de redes é, principalmente, uma mudança no perfil demográfico da população do planeta, que tem envelhecido. O que se observa principalmente nos países de primeiro mundo é que as doenças infecciosas que representavam, epidemiologicamente, a principal causa de morte no início do século $X X$, já estão relativamente controladas. Eu digo relativamente, exatamente porque na nossa dificuldade de lidar com problemas sistêmicos, temos também dificuldade de enxergarmos a natureza cíclica destes problemas, uma característica fundamental da complexidade. Há sempre um retorno possível de alguns destes problemas que na nossa vã ingenuidade postulamos ter superado de forma definitiva.

O fato é que as pessoas hoje vivem mais, mas, também, têm mais propensão ao

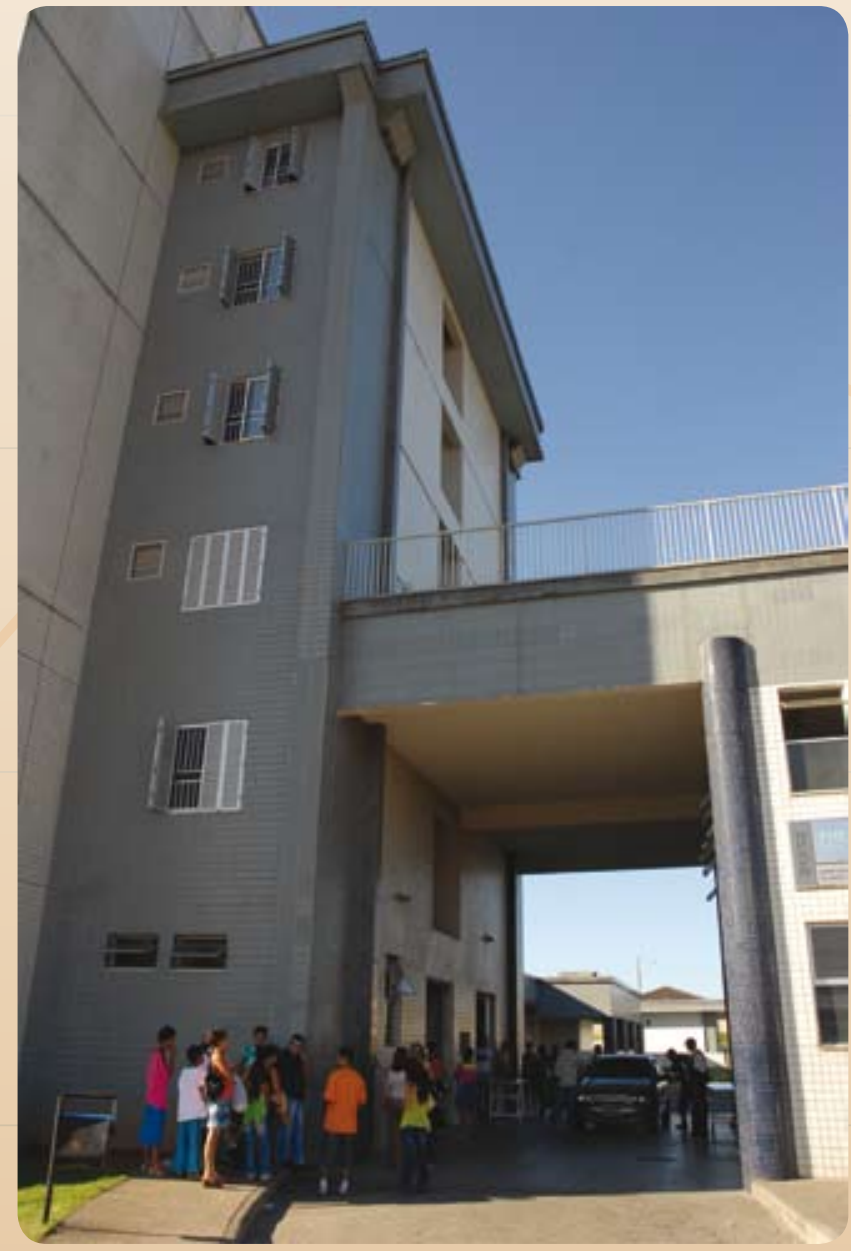
desenvolvimento de doenças crônicas, como o diabetes, as doenças cardiovasculares e o câncer. O modelo de organização dos sistemas de saúde atual não responde adequadamente a estas doenças, pois, são focados em estruturas separadas e com predominância das de maior tecnologia mecânica, numa herança da metáfora da ciência clássica. O resultado final deste modelo tem sido desastroso. Uma das manifestações deste desastre é o excesso de pessoas nas portas de atendimento de urgências. Este é hoje o maior sintoma de um grave problema que os sistemas de saúde no mundo vivenciam: um modelo equivocado baseado em premissas extemporâneas. A solução para este problema não é pontual ou local, mas sistêmica. As pessoas que se espremem para tentar entrar num pronto socorro apresentam, em $90 \%$ das vezes, agudizações de problemas crônicos. Este problema não é privilégio nosso. Como dissemos no início do artigo, a tentativa dos países de primeiro mundo de inverter o modelo de seu sistema de saúde na década de 90 , coincide com o surgimento dos primeiros protocolos de triagem de urgência sistematizados. 
Os 4 principais protocolos de triagem nas urgências surgem "coincidentemente"neste período. São eles: Australiano, Manchester (Inglês), Canadense e ESI (americano). Interessantemente, apresentam algumas coincidências nos seus princípios, diferenciando radicalmente no modelo de entrada da queixa e num deles (ESI) na necessidade de recursos influindo na decisão final. O quadro abaixo compara estes modelos:

\begin{tabular}{|l|c|c|c|c|c|}
\hline CARACTERÍSTICAS & ATS & CTAS & MTS & ESI & MAT \\
\hline Escala de 5 Níveis & SIM & SIM & SIM & SIM & SIM \\
\hline Utilização universal no país & SIM & SIM & SIM & NÃO & SIM \\
\hline Baseado em categorias de sintomas & NÃO & NÃO & SIM & NÃO & SIM \\
\hline Baseado em discriminantes chave & SIM & NÃO & SIM & SIM & SIM \\
\hline Baseado em algoritmos clínicos & NÃO & NÃO & SIM & SIM & SIM \\
\hline Baseados em escalas de urgência pré-definidas & SIM & SIM & NÃO & NÃO & SIM \\
\hline Formato eletrônico (Informatizado) & NÃO & NÃO & SIM & NÃO & SIM \\
\hline
\end{tabular}

Fonte: J.Gimenez: Emergências 2003;15:165-174

- Modelo Australiano (Australasian Triage Scale - ATS) - Foi o pioneiro e usa tempos de espera de acordo com gravidade;

- Modelo Canadense (Canadian Triage Acuity Scale - CTAS) - Muito semelhante ao modelo australiano, é muito mais complexo e está em uso em grande parte do sistema canadense. O mecanismo de entrada é uma situação pré-definida;

- Modelo de Manchester (Manchester Triage System - MTS) - Trabalha com algoritmos e determinantes, associados a tempos de espera simbolizados por cor. Está sistematizado em vários países da Europa. O mecanismo de entrada é uma queixa;

- Modelo Americano (Emergency Severity Index - ESI) - Trabalha com um único algoritmo que foca mais na necessidade de recursos para o atendimento. Não é usado em todo o país;

- Modelo de Andorra (Model Andorrà del Trialge - MAT) - Baseia-se em sintomas, discriminantes e algoritmos mas é de uso complexo e demorado(tempo médio de 8 minutos)

Mas a adoção de um protocolo de triagem resolve o problema? Obviamente que não. Ele é um poderoso instrumento para iniciar um processo de gestão em estruturas naturalmente desorganizadas, como os hospitais de urgência e isto é apontado na literatura mundial. Não existe mais possibilidade de trabalhar com atenção de urgências sem um sistema de triagem ou classificação de risco e é demonstrado, também, que alguns deles, principalmente o Manchester que é o mais usado no mundo hoje, se relacionam com a previsão de mortalidade nas primeiras 24 horas e na necessidade de internação. No entanto, se estas estruturas ainda trabalham de forma isolada e não se organizam com os outros pontos de atenção, o resultado da implantação de um protocolo destes é menor do que seu potencial. Eis um fato que temos observado, tanto aqui no Brasil, quanto no exterior. Se não trabalharmos em rede os problemas não cessam. Recentemente, o Dr. Kevin Macway, um dos autores do Protocolo de Manchester, que hoje faz parte das recomendações do Advanced Life Suport Group, assim como o ATLS e ACLS, largamente adotados no Brasil, observou que vem aumentando anualmente a procura das urgências no Reino Unido e principalmente por pacientes que apresentavam baixo risco após a triagem. Os problemas, aqui no Brasil, são similares, assim como nos Estados Unidos, no Japão, que também está adotando o Protocolo de Manchester e em toda a Europa. Qual é hoje o grande consenso de quem trabalha nos sistemas de atenção às urgências? A solução para as urgências está na mudança do modelo de atenção do sistema de saúde. Isto significa adotar radicalmente um modelo que privilegie a atenção primária, não a atenção"básica" praticada em nosso país,que se configura como um pacote, uma cesta básica de serviços não eficazes, mas, uma nova atenção primária, resolutiva e forte que se torna o centro de comunicação e o alicerce mais importante de uma rede integrada de serviços de saúde. Esta é a base de outra grande revolução 
seguindo o que outras áreas da atividade humana vêm fazendo de forma mais rápida: estruturação em rede. As redes são sistemas complexos, estudados hoje em matérias tão diversas como a física e a economia e que têm características interessantes. O que se sabe hoje é que a própria natureza e a maioria dos fenômenos orgânicos e econômicos se estruturam em uma grande e complexa rede de conexões. $O$ que alguns estudos mostram como propriedades de um sistema em rede?

1)Auto-organização: $O$ processo de organização em redes é um processo natural, mas que leva tempo. $O$ que aproxima os vários componentes de uma rede não é a sua semelhança, mas a existência de padrões comuns. Em redes sociais isto pode ser induzido com a criação de lideranças, templates, mas principalmente de uma linguagem padrão;

2)As redes de comunicação se diferenciam por 2 modelos: Small world e Scale free. A diferença entre estas redes é que na Scale free, muitas vezes, um ponto (ou hub) terá passagem de informações superior aos outros. Na natureza, isto é aleatório e emergente. As redes de urgência têm este padrão, mas, podemos obter a diminuição da aleatoriedade, por exemplo, pelo comando de uma central de regulação;

3)Feedback: As mudanças numa rede ocorrem através deste fenômeno, que pode ser negativo na tentativa de se manter algum padrão ou positivo caso suplante e transforme o modelo anterior de organização.

Este é o fato que nos mostra a necessidade de estabelecer padrões que unam os pontos de atenção numa rede de serviços de saúde. Estes padrões, que são um pouco o software de uma rede de saúde, na nossa proposta, se estabelecem numa tentativa de estratificação do risco numa determinada variação temporal, buscando estabelecer as conexões entre os pontos de atenção e a logística (transporte, central de regulação etc..). Um exemplo é um guideline de diabetes que estratifica o risco de uma população e determina o fluxo no tempo e espaço, por indivíduo, por uma rede de serviços de saúde e também possibilita mensurar a evolução deste processo. Neste caso, o risco é estratificado a médio e longo prazo. No caso de um quadro agudo, o risco é estratificado na necessidade do tempo de espera pelo primeiro olhar médico e se configura também numa previsão de mortalidade nas primeiras horas, assim como numa previsão da necessidade de recursos para aquela situação. Se esta estratificação for usada como linguagem padrão em todos os níveis de atenção e pela logística do sistema, no caso específico dos pacientes agudos, estará sendo estabelecido um fluxo razoavelmente direcionado, reduzindo a imprevisibilidade das situações de urgência. Por isto, temos recomendado que um protocolo de triagem ou classificação de risco deveria ser único numa rede integrada de serviços de saúde, pois, aí sim, estaremos utilizando todo o potencial deste instrumento.

Para finalizar, temos a convicção de que o protocolo de classificação de risco, seja qual for, não é uma panacéia para os problemas de urgência, mas é um instrumento de gestão muito potente, assim como é uma linguagem que reduz significativamente a variabilidade numa rede complexa, como a rede de urgência. Em Minas Gerais, adotamos o que é hoje o protocolo mais estudado e mais usado no mundo atualmente, o de Manchester, sendo o mais seguro quando bem aplicado. Para isto existe a formação dos executantes, pois o protocolo segue fluxogramas bem definidos e não inventados pela cabeça do executante, tornando possível a auditoria de forma estruturada. Assim, todos os serviços que usam o protocolo devem manter o padrão de utilização deste, conforme seus princípios e estabelecer benchmarking entre os mesmos. Isto gera um processo de qualidade e, no momento pós-triagem, uma possibilidade de estabelecer fluxos pactuados e mais seguros para as pessoas, diferentemente da variabilidade caótica e pouco eficaz que se vê nos pontos de atenção às urgências nos sistemas de saúde atuais. 


\section{REFERÊNCIAS BIBLIOGRÁFICAS}

1) ALETRAS, V.; JONES, A.; SHELDON, T. A. Economies of scale and scope. In: FERGUSON, B., SHELDON, T.A; POSNETT, J. Concentration and choice in health care. London, Financial Times Healthcare, 1997.

2) BANTA, H. D.; BOS, M. The relation between quantity and quality with coronary artery bypass surgery. Health Policy, 18: 1-10, 1991.

3) BUNKER, J. P., LUFT, H. S.; ENTHOVEN, A. Should surgery be regionalised? Surgical Clinics of North America, 62: 657-668, 1982.

4) FERGUSON, B.; TREVOR, A. S.; POSNETT, J. Introduction. In: FERGUSON, B., TREVOR, A S; POSNETT, J. (Editors) Concentration and choice in healthcare. London, FT Healthcare, 1997.

5) BRASIL. Ministério da Saúde A regionalização da saúde: versão preliminar para discussão interna. Brasília, mimeo, 2004.

6) NORONHA, J. C. Avaliação da relação entre volume de procedimentos e a qualidade do cuidado: o caso da cirurgia coronariana. Cadernos de Saúde Pública, 19: 1781-1789, 2003.

7) Guidelines for Essential Trauma Care - WHO 2004);

8) GIMENEZ, J. Emergências 2003;15:165-174.

9) CAN, J. Emerg. Medicine2006; 8(s); 148-57- Thimothy Cooke.

10) COOKE M. W.; JINKS, S. J. Does the Manchester triage system detect the critically ill? Accid Emerg. Medicine 1999.

11) SUBBE C. P.; SLATER, A; MENON, D; GEMMEL, L. Emerg Validation of physiological scoring system in the A\& $E$ department; Med Journal, 2006.

12) SPEAK, D.; TEECE, S.; MACKWAY, J. K. Detecting High risk patients with Chest pain, Emergency nurse 2003.

13) Manchester (MTS) more than a triage system? Studying it's association with death and admission in a large Portuguese Hospital-bmj (no prelo).

14) EMERGENCY MEDICINE JOURNAL. Reliability and validity of Manchester Triage system in a general emergency department patient population in the netherlands: results of a simulation study. 2008

15) MATIAS Carla; OLIVEIRA Ricardo; DUARTE Rita; et al. Triagem de Manchester nas Síndromes Coronárias Agudas = The Manchester triage System in Acute Coronary Syndromes; Revista Portuguesa de Cardiologia ISSN 0870-2551 Source / Source2008, vol. 27, no2, pp. 205-216

16) ROUKENA, Jolt; LEI, Johan; et al. Emergency care: prospective observational study Manchester triage system in paediatric:10.1136/bmj.a1501BMJ 2008;337.

17) MELINDA, Lyons; RUTH, Brown; ROBERT, Wears. Factors that affect the flow of patient/s through triage. Postgraduate Medical Centre, Cambridge University Hospitals NHS Foundation Trust, University Department of Anaesthesia, Addenbrooke's Hospital, Cambridge, UK.

18) EMERGENCE MEDICINY JOURNAL. Accident and Emergency Department, St Mary's Hospital, London, UK Clinical Safety Research Unit, Department of Surgical Oncology and Technology, Imperial College, St Mary's Hospital, London, UK 2007;24:78-85; doi:10.1136/emj.2006.036764

19) MIRJAM, Van Veen; HENRIETTE, A. Moll. Validity of triage systems: use a correct outcome measure. Emerg Med $\mathrm{J}$ 02/09/2008.

20) EMERGENCE MED. JOURNAL. Don't throw triage out with the bathwater J Windle1, K Mackway-Jones1 Department of Emergency Medicine, Hope Hospital, Salford/Salford University, UK. Department of Emergency Medicine, Manchester Royal Infirmary, UK 2003; 20:119-120

21) EMERGENCE MED. JOURNAL. Reliability and validity of the Manchester Triage System in a general emergency department patient population in the Netherlands: results of a simulation study. 2008 Jul. I van der Wulp, M E van Baar, A J P Schrijvers Ms I van der Wulp, Julius Center for Health Sciences and Primary Care, UMC Utrecht, P O Box 85500, 3508 GA Utrecht, The Netherlands; i.vanderwulp@umcutrecht.nl.

22) ANESTESIST. Manchester triage system: Process optimization in the interdisci

23) ESI-Version 4

24) CASTELLS, M. - A sociedade em rede. São Paulo, Paz e Terra, Volume I, $4^{a}$ ed., 2000.

25) CENTER FOR DISEASE CONTROL AND PREVENTION - Framework for program evaluation in public health. MMWR 48, N'RR-11, 1999.

26) DONABEDIAN, A. Benefits in medical care programs. Cambridge, Harvard University Press, 1976.

27) INSTITUTE OF MEDICINE - Defining primary care: an interim report. Washington, The National Academy Press, 1994.

28) JOINT COMISSION ON ACCREDITATION OF HEALTHCARE ORGANIZATIONS - Comprehensive accreditation manual for integrated delivery systems. Oakbrook Terrace, Illinois, Joint Commission Resources, 2004. 
29) MENDES, E.V. Uma agenda para a saúde. São Paulo, Ed. Hucitec, $2^{a}$ ed., 1999.

30) MENDES, E.V. Os grandes dilemas do SUS. Salvador, Casa da Qualidade, Tomo II, 2001.

31) MENDES, E.V. Os sistemas de serviços de saúde: o que os gestores deveriam saber sobre essas organizações complexas. Fortaleza, Escola de Saúde Pública do Ceará, 2002a.

32) MENDES, E.V. A atenção primária à saúde no SUS. Fortaleza, Escola de Saúde Pública do Ceará, 2002b.

33) MENDES, E.V. Os modelos de atenção à saúde. Belo Horizonte, mimeo, 2007a.

34) MENDES, E.V. As redes de atenção à saúde no Brasil: o caso de Curitiba. Washington, Organização Pan-Americana da Saúde, 2007b.

35) SHIMAZAKI, M.E. Oficina de rede atenção às urgências e emergências. Belo Horizonte, Secretaria de Estado de Saúde de Minas Gerais, 2007.

36) VARGAS, I.L. Algunas conclusiones y retos de futuro para las OSI en Cataluña. In: VÁZQUEZ, M.L.N. \& VARGAS, I.L. - Organizaciones sanitarias integradas: un estudio de casos. Barcelona, Consorci Hospitalari de Catalunya, 2007.

37) VÁZQUEZ, M.L.N. et al. Organizaciones sanitarias integradas: una guía para el análisis. Rev. Esp. Salud Pública, 79: 633-643, 2005.

38) VÁZQUEZ, M.L.N. \& VARGAS, I.L. Redes integradas de servicios de salud: solución o problema? Ciencias de la Salud, 4: 5-9, 2006.

39) CTAS. The Canadian Emergency Department Triage \& Acuity Scale

40) ATS. Australasian Triage Scale

41) JONES, K M; Manchester Triage System (MTS) Second edition BMJ

42) ATLS. Advanced Trauma Life Suport. Eighth edition

43) ACLS. Advanced Cardiac Life Suport. 2005

44) MORIN, E. O pensar complexo- Editora UNB

45) Centro teórico de física computacional- União Européia- www.ctfc.cii.fc.ul.pt/PRISMA- Redes Complexas 